oceanography with no prior knowledge of fluids, this can be quite useful even if much of it is readily available elsewhere.

Pedlosky on the other hand is suitable for more advanced graduate students and specialists. Almost everyone in this category is bound to find something of interest in this intensive book each time he opens it, be it for casual reading or reference. The scope of the book could have been more catholic but the treatment of his chosen material is generally very thorough, one notable exception being equatorial dynamics, an important aspect of modern thinking which gets only marginal coverage in the last chapter (and none at all from von Schwind). The mid-latitude quasi-geostrophic potential vorticity equation is well explained and there is a good section on instability theory. The presentation is mathematically, rather than physically orientated but Pedlosky is a text one can recommend to all serious students of GFD.

D. Anderson is a Lecturer in Atmospheric Dynamics at Oxford University.

\section{Unmathematical air}

\section{John Green}

\author{
Atmospheric Physics. By J. V. Iribarne \\ and H.-R. Cho. Pp.212. (Reidel: 1980.) \\ Dfl. $40, \$ 15.95$.
}

ANOTHER text that is said to require only elementary mathematics - in this case to understand the terrestrial atmosphere. I must confess a bias. To my mind no book on physics should use more complex mathematics than is needed to make its point. Conversely, no text should go out of its way to avoid some essential mathematics because the reader may not understand it. He had better be made aware of his deficiency and of the intellectual benefit that will result from being able to understand the mathematical part of an argument.

The authors identify the need for a survey of the physics of the terrestrial atmosphere, suitable for students having completed only one year of a course in general physics. They give a general description of the structure of the atmosphere, atmospheric chemistry, radiation, thermodynamics, cloud physics, and atmospheric electricity and dynamics. Each topic is treated sympathetically but briefly. At the end of each chapter there are questions and problems designed to aid the learning process, and hints on solutions at the end of the book. Diagrams are mostly well drawn but the writing is occasionally careless.

The philosophy of presenting material without recourse to more than elementary mathematics is admirable. Execution is less so. The student is given the appropriate formula with only the most general guidance to the most comprehensive monographs where, I believe, he will find the derivation very difficult to follow. More detailed reference to a text of intermediate standard would help. I think it is useful to comment that a stated formula contains some of the variables you would expect and has a plausible functional form, besides giving a detailed

reference to a succinct derivation in the same notation.

This quibble apart, our poor, unfortunate, non-mathematical person will find this a useful, interesting and balanced survey of atmospheric physics.

John Green is Reader in Physical Climatology and teaches a course in atmospheric physics at Imperial College of Science and Technology, University of London.

\title{
Star of the lecture theatre
}

\section{Stephen P. Maran}

Discovering the Universe. By Charles E. Long. Pp.511. (Harper \& Row: 1980.) Pbk $\$ 16.50$. Dynamic Astronomy. 3rd Edn. By Robert T. Dixon. Pp.523. (Prentice-Hall: 1980.) Pbk £11, \$16.95. Teacher's manual also available.

AT A university in southern California, 500 undergraduates in a long, narrow auditorium observe a professor, dimly visible at centre stage, microphone around the neck, lecture on black holes, the Big Bang and life in space. At an East Coast university, a comparable throng files into two adjacent auditoria as the dividing wall between the rooms is electrically retracted; at the same time a large turntable at the front of one of the halls slowly rotates away the entomology instructor who has just finished a lecture. Into view spins the astronomer with demonstration table, microphone and piles of computer-graded examinations that are ready for return to the students. As the lecture begins and the lights fade away, a coloured photograph of a spiral galaxy appears overhead, projected in duplicate to provide identical side-byside images for the benefit of those sitting at far left and far right in the wings of the assembly.

As the elementary, non-mathematical course in astronomy has become so popular in North America, perhaps 50 competing texts have appeared. The latest, Discovering the Universe, is alternately thoughtful and chatty, with many apropos anecdotes and numerous, seeminglyscrawled sketches that are as effective as the usual formal diagrams. At its best (taken on its own terms), Discovering introduces the unwary to general relativity with the aid of two inquisitive ants (Einstant and Newtant), or describes Mercury as seen by Mariner 10. In the latter case, only a few details are given, but much effort then follows in explaining what one might experience on a visit to the planet. The explanation is clear enough that the student will understand why each prospective phenomenon is anticipated. Marring the otherwise accurate text, however, are erroneous statements (each of which I have seen before) to the effect that Uhuru discovered Cygnus X-1 and that the optical pulsar in the Crab Nebula was discovered on the famous Lick Observatory rotating-shutter photographs. Those photos were obtained well after telegrams announced the discovery of the optical pulses at Steward Observatory, their confirmation at McDonald Observatory, and their identification with a specific star - the pulsar - at Kitt Peak National Observatory.

Dynamic Astronomy, here in a third edition, is distinguished by "flip pages", which bear in the margins sequences of diagrams that can be made to spring into life. Riffle them and you will observe Halley's Comet in retrograde motion, tail growing as it approaches the Sun, then shrinking as the comet hurtles back in to the far reaches of the Solar System. Other sequences depict planetary, stellar and star cluster motions. Better yet, try turning the same pages slowly and you will find besides the diagrams a well-polished text, familiar by now to the students "in over two hundred colleges and universities". Yet improvements are still to be made. A section on "Observing Meteors" gives the wrong explanation for why more meteors are observed after midnight than before. Contrary to Fig.9-15, globular clusters are strongly concentrated to the centre of the Galaxy although they are more conspicuous in the halo. The sources of the gamma-ray bursts discovered by Klebesadel with data from the Vela satellites are confused with the very different "X-ray bursters" studied by Grindlay. Less significantly, although surprising in a mature text, the names of astronomers often are spelled wrong, including those of Olbers, Jastrow, Solomon and Maran. Somehow, when the reviewer's name is misspelled, it always is noticed.

Stephen P. Maran is at the Laboratory for Astronomy and Solar Physics, NASAGoddard Space Flight Center, Greenbelt, Maryland.

The Institute of Biology has published a Handbook for the Animal Licence Holder, edited by H.V. Wyatt, intended particularly for newcomers to laboratory animal work. The Handbook is available from 41 Queen's Gate, London SW75HU, price $£ 2.50$ or $£ 2$ for five or more copies. 